\title{
The Inferior Frontal Gyrus and Phonological Processing: An Investigation using r'TMS
}

\author{
Philip Nixon ${ }^{1}$, Jenia Lazarova ${ }^{2}$, Iona Hodinott-Hill ${ }^{1}$, Patricia Gough ${ }^{1}$, \\ and Richard Passingham ${ }^{1}$
}

\begin{abstract}
Repetitive transcranial magnetic stimulation (rTMS) offers a powerful new technique for investigating the distinct contributions of the cortical language areas. We have used this method to examine the role of the left inferior frontal gyrus (IFG) in phonological processing and verbal working memory. Functional neuroimaging studies have implicated the posterior part of the left IFG in both phonological decision making and subvocal rehearsal mechanisms, but imaging is a correlational method and it is therefore necessary to determine whether this region is essential for such processes. In this paper we present the results of two experiments in which rTMS was applied over the frontal operculum while subjects performed a delayed phonological matching task. We compared the effects of disrupting this area either during the delay (memory) phase or at the response (decision) phase of the
\end{abstract}

\section{INTRODUCTION}

Transcranial magnetic stimulation (TMS) provides an effective new method for investigating cognitive functions in human subjects (see Jahanshahi \& Rothwell, 2000, for a review). TMS can be used to test the functional necessity of the cortical activations seen in functional neuroimaging studies on human subjects.

Neuroimaging techniques have demonstrated widespread left-sided ventral frontal activations in relation to many forms of linguistic processing (Price, Indefrey, \& Turennout, 1999). Hemodynamic changes that have been attributed to the phonological encoding of words into articulatory plans have been reported during repetition of heard words (Price et al., 1996), silent repetition of nonwords (Warburton et al., 1996), phonemic fluency tasks (Paulesu et al., 1997), and when making phonological judgements (Poldrack et al., 1999; Demonet et al., 1992; Zattore, Evans, Meyer, \& Gjedde, 1992). All these activations were localized to a region that included the posterior (opercular) division of Broca's area (BA 44) and tissue in the descending limb of the precentral sulcus (BA 6). However, the fact that these areas are shown to be more

\footnotetext{
${ }^{1}$ University of Oxford, ${ }^{2}$ Whittier College
}

task. Delivered at a time when subjects were required to remember the sound of a visually presented word, rTMS impaired the accuracy with which they subsequently performed the task. However, when delivered later in the trial, as the subjects compared the remembered word with a given pseudoword, rTMS did not impair accuracy. Performance by the same subjects on a control task that required the processing of nonverbal visual stimuli was unaffected by the rTMS. Similarly, performance on both tasks was unaffected by rTMS delivered over a more anterior site (pars triangularis). We conclude that the opercular region of the IFG is necessary for the normal operation of phonologically based working memory mechanisms. Furthermore, this study shows that rTMS can shed further light on the precise role of cortical language areas in humans.

active does not establish that they are necessary for phonological processing.

TMS has been used to examine the roles of the temporal lobe language areas in picture-naming tasks (Stewart, Meyer, Frith, \& Rothwell, 2001; Topper, Mottaghy, Brugmann, Noth, \& Huber, 1998). Studies of frontal lobe language functions have remained largely restricted to frontal regions outside Broca's area; these have demonstrated that TMS can be used to disrupt verbal working memory (WM) (Mottaghy et al., 2000; Mottaghy, Doring, Muller-Gartner, Topper, \& Krause, 2002) and verb generation (Shapiro, Pascual-Leone, Mottaghy, Gangitano, \& Caramazza, 2001). TMS studies attempting to reproduce some of the production deficits associated with Broca's aphasia have had limited success (Stewart, Walsh, Frith, \& Rothwell, 2001; Flitman et al., 1998; Epstein et al., 1996, 1999). Speech disruption can be obtained using repetitive trains of TMS pulses at levels well above the threshold for eliciting movements of the digits when applied over motor cortex. However, the sites at which such disruption generally occurs tend not to lie below the inferior frontal sulcus where Broca's area is located (Stewart, Meyer, et al., 2001; Bartres-Faz et al., 1999). Furthermore, the relatively severe speech production deficits associated with Broca's aphasia may to some degree result from damage lying outside this area, espe- 
cially to the white matter lying below the cortical surface (Willmes \& Poeck, 1993; Signoret, Castaigne, Lhermitte, Abelanet, \& Lavorel, 1984). TMS typically affects a region of cortex no larger than $1-2 \mathrm{~cm}$ in diameter (Walsh \& Cowey, 2000) and might not therefore be expected to reproduce the clinical effects of lesions resulting from major arterial strokes or the removal of large tumors.

In the study presented here, we examine whether or not it is possible to interfere with phonological processing by applying short, high-frequency trains of TMS pulses over Broca's area. We conducted two experiments in which TMS was applied over this region while normal subjects performed a delayed phonological matching task. The design of the first experiment allowed us to contrast the effects of the TMS on verbal WM and on decision-making processes. The second experiment compared the effects of applying TMS separately to the anterior (BA 45) and posterior (BA 44) divisions of Broca's area. In order to control for any nonspecific effects of TMS that might affect performance on this task, the results were compared with the effects of TMS on a nonverbal visual matching task. Because the TMS in these experiments caused reaction time (RT) decreases, a third experiment was conducted using sham TMS to test whether or not these decreases were caused by effects other than the direct magnetic stimulation of the brain.

\section{RESULTS}

\section{Experiment 1: Contrasting Working Memory and Decision Processes}

In the first experiment we compared the effects of applying TMS either while subjects remembered the sound of a word they had seen, or when they were required to match this word with the sound of a subsequently presented pseudoword (homophone task). The control task differed from this task only in the nature of the stimuli used (Korean letters). Subjects were thus required to remember and subsequently match visual symbols that lacked (to non-Korean speakers) any phonological representation. The experiment was based on a $2 \times 3$ block design with Task (phonological, visual) and TMS (none, delay, response) as manipulated factors. We were thus able to compare the effects of applying TMS during the delay period (1 sec), while subjects held cue information in memory, with TMS applied later in the trial as subjects compared the memorized cue with a second cue and implemented their response.

The two tasks were presented consecutively with task order, but not TMS onset, counterbalanced across subjects. Short trains of TMS were applied over the pars opercularis region of the left hemisphere at approximately $120 \%$ of the subject's active motor threshold. The site was estimated with respect to the physiologically determined hand representation of motor cortex. Be- cause movements of the temporal muscle/jaw are commonly elicited by TMS over the ventral part of the frontal lobes, the subjects were instructed not to use overt speech during performance of the homophone task. Covert speech has been shown to elicit strong hemodynamic responses in the posterior division of Broca's area (BA 44), near its border with premotor cortex (BA 6) (Grezes \& Decety, 2001; Warburton et al., 1996; Paulesu, Frith, \& Frackowiak, 1993). This region was the target for TMS stimulation in this experiment (see Figure 5C).

\section{Results and Discussion}

On non-TMS trials, subjects generally performed both tasks with a high degree of accuracy. For the homophone task the mean error rate (ER) was 0.83 trials and for the control task it was 0.67 trials. The mean RTs on non-TMS trials were $831 \mathrm{msec}$ (homophone task) and 584 msec (control task).

When TMS was applied during the delay it significantly impaired accuracy on the homophone task, $t(5)=2.70$, $p<.05$, but not on the control task, $t(5)=.10$, ns (Figure 1A). The differential effect on the two tasks was also significant, $t(5)=3.16, p<.05$. TMS applied over the same site at the time of the response was also accompanied by an increase in errors but this did not reach significance because of the greater between-subject variability (Figure 1B).

On both tasks, there were significant decreases in RT (Figure 1), both when TMS was applied in the delay, homophones: $t(5)=-6.29, p<.01$; control: $t(5)=$ $-6.74, p<.01$, and at response, homophones: $t(5)=$ $-3.36, p<.05$; control: $t(5)=-4.65, p<.01$. However, there were no significant differences between the tasks on this measure, delay: $t(5)=-2.15$, ns; response: $t(5)=-1.09, n s$.

The results clearly show that TMS had a greater effect on a task that required processing of verbal material than on a similar task that required only visual matching. The stimulation was more effective when applied during the delay period between presentation of the verbal stimuli. This is consistent with the evidence that the posterior inferior frontal gyrus (IFG) is activated during the maintenance of phonological representations (Paulesu et al., 1993). It also showed that this activity is necessary for accurate performance.

Facilitatory effects on RT were seen for all TMS blocks and are of interest for two reasons. First, TMS is generally considered to disrupt processing in higher cortical areas (Walsh \& Cowey, 2000; Pascual-Leone et al., 1999), and this is indexed by measurable increases in RT on cognitive tasks. However, this outcome can be counterbalanced by a facilitation of responses resulting from intersensory facilitation effects (Hershenson, 1963). We measured the magnitude of this effect in Experiment 3 by testing with sham stimulation. The second concern is that given the RT decreases, it could be argued that they 
Figure 1. Results of

Experiment 1. Changes in percent error rate (\%ER) and RT for TMS versus non-TMS trials. (A) When rTMS was applied over the posterior (opercular) site during the delay period the mean increase in \%ER was much larger for homophone task trials (black bars) than for control task trials (gray bars). There was a similar decrease in RT for both tasks at this site (lower graph). (B) When TMS was applied at the decision phase of each task, there was some evidence of an increase in \%ER for the homophone task but the variance of the mean was very high. As for stimulation applied during the delay, there was a strong facilitation of RT for both tasks (lower graph). The standard error of the means is shown by the thin bars.

$* p<.05, * * p<.01$

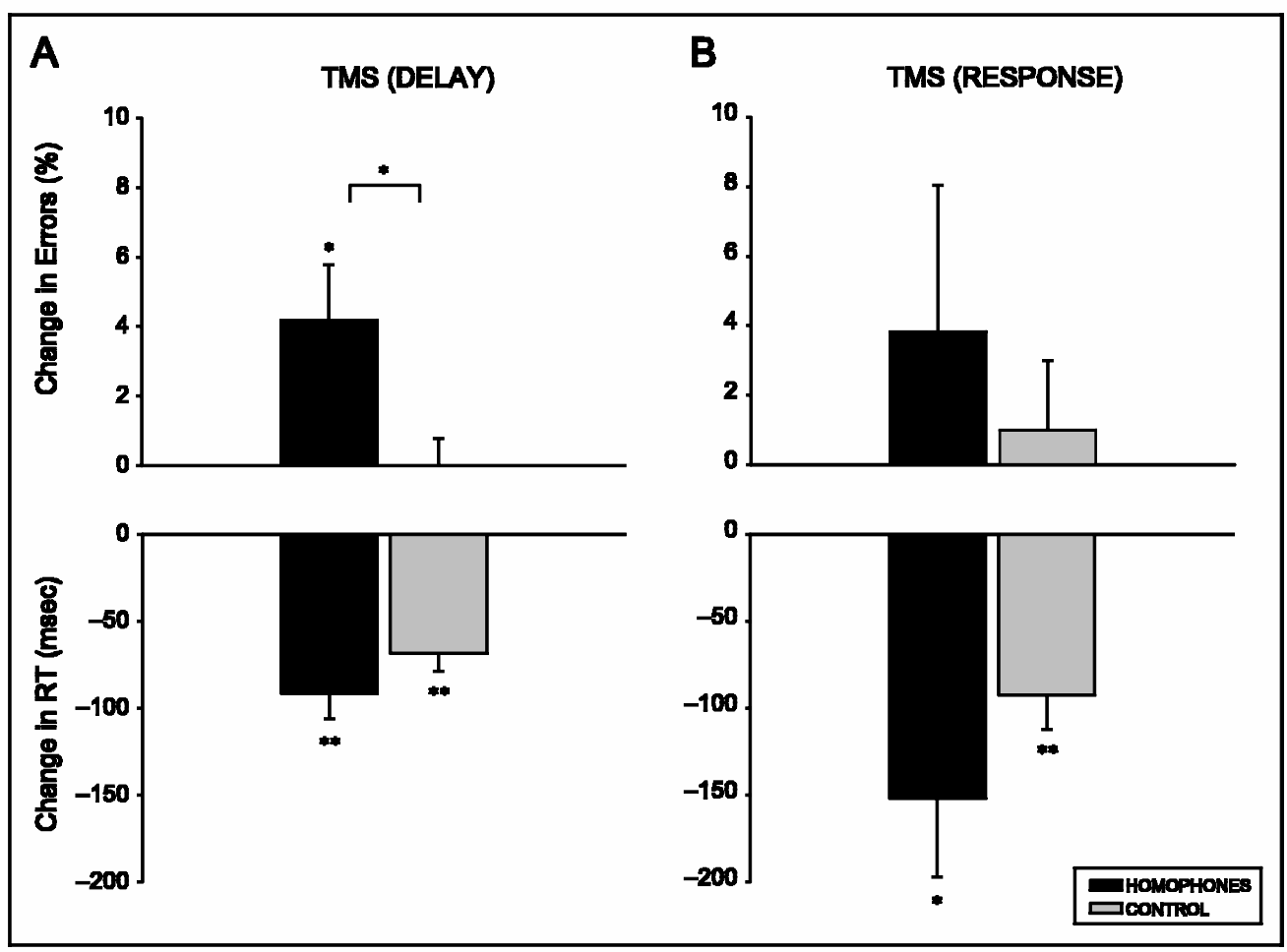

may have had an adverse impact on the accuracy with which subjects performed the task (due to a speed-error trade-off). However, it can be seen (Figure 1A) that while there were decreases in RT on both tasks, accuracy was impaired only on the verbal task.

\section{Experiment 2: Contrasting Pars Opercularis and Pars Triangularis}

In this experiment we sought to replicate the main finding of the previous experiment, that is, the detrimental effect of TMS on verbal WM, and to compare this with the effects of TMS over the pars triangularis region of Broca's area. The design of the current experiment improves on the previous one because of the introduction of a frameless stereotaxy imaging system into the laboratory. This permits a more precise neuroanatomical localization of the stimulation target sites based on structural MRIs of the subjects participating in the experiment. The system was used to enable a better localization of the pars opercularis region of the IFG than had been achieved in Experiment 1 and to allow a comparison of stimulation applied to this area with stimulation applied to the adjacent, pars triangularis, region. There is some debate as to whether this latter region has a dissociable role in semantic, rather than phonological, processing (McDermott, Petersen, Watson, \& Ojemann, 2003; Poldrack et al., 1999; Price et al., 1999; Demonet et al., 1992). We hypothesized that TMS applied here would be less likely to impair verbal WM for phonological representations of words and would provide a suitable control site for the uncomfortable facial muscle twitches associated with ventral frontal repetitive TMS (rTMS). The design of this experiment thus differs from the previous one in that the manipulated factor TMS now refers to site (i.e., none, anterior, posterior) rather than onset, with TMS being applied exclusively during the delay period. A further modification was made by increasing the within-trial delay period to $2000 \mathrm{msec}$ to increase the WM load, with the TMS onset starting at $1500 \mathrm{msec}$ and lasting $500 \mathrm{msec}$.

\section{Results and Discussion}

The results for stimulation over the posterior site were similar to those of Experiment 1. TMS applied during the delay period impaired accuracy for the phonological task, $t(5)=2.74, p<.05$, but not for the control task (Figure 2A). The magnitude of the impairment was similar to that observed in the first experiment despite the increase in delay length in the current design. The differential effects of TMS at this site on the two tasks were also significant, $t(5)=3.61, p<.05$, but there was no difference in its effects over the anterior site, $t(3)=$ 1.00 , ns (Figure 2B). In addition, a $2 \times 3$ ANOVA with Task (phonological, visual) and TMS (none, posterior, anterior) as independent factors confirmed a significant interaction, $F(2,6)=5.4, p<.05)$.

Significant decreases in RT were seen for the homophone task when TMS was applied over the opercular region, $t(5)=-3.50, p<.05$, and similarly for the control task when TMS was applied over the triangular region, $t(3)=-5.51, p<.05$, but there were no 
Figure 2. Results of Experiment 2. Changes in percent error rate (\%ER) and RT for TMS versus non-TMS trials. (A) The effects of rTMS applied over pars opercularis during the delay period were reversed for homophone task trials (black bars) as compared to control task trials (gray bars). There was a marked decrease in RT for homophones, as seen in Experiment 1. (B) However, when TMS was applied over pars triangularis, there were no significant changes in \%ER seen on either task. As before, there was a marked facilitation of RT on both tasks (lower graph). The standard error of the means is shown by the thin bars. $* p<.05$.

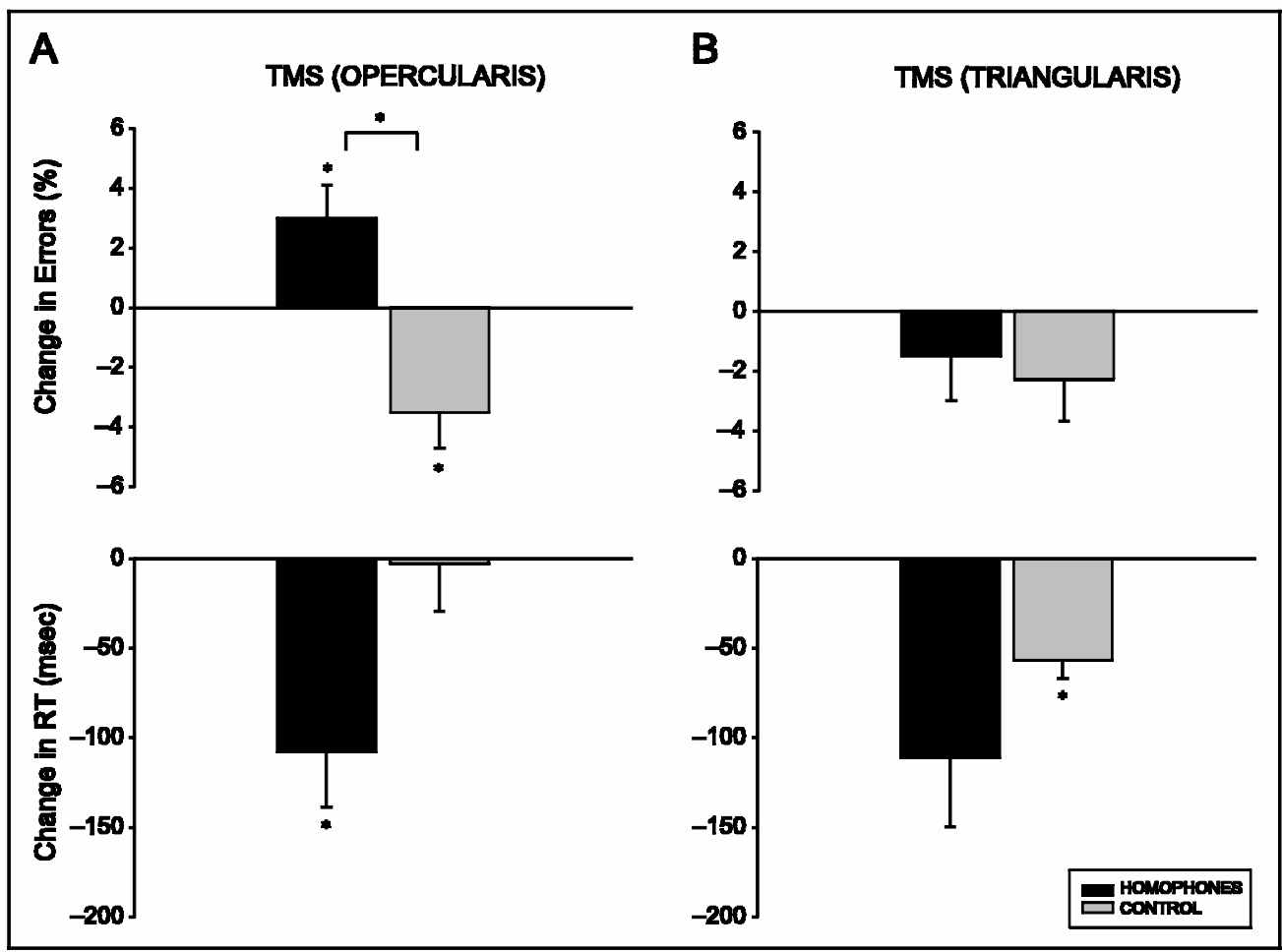

significant differences between tasks at either site (Figure 5) and no interactions, $F(2,6)=1.39$, ns.

The results of this experiment confirm the findings of Experiment 1 for TMS applied over the posterior (pars opercularis) division of the IFG during the delay period. The absence of any increase in ER for TMS over the anterior (pars triangularis) region suggests this region is not necessary for maintaining phonological representations of words over time. Yet neuroimaging studies have generally reported that both regions are active during phonological and during semantic processing (Devlin, Matthews, \& Rushworth, 2003; Paulesu et al., 1997; Demonet et al., 1992). It is possible that both regions are coactivated because semantic processing invokes implicit phonological representations of verbal stimuli, and vice versa. (Devlin et al., 2003; Poldrack et al., 1999; Price et al., 1999). However, the results of Experiment 2 show that disruption of activity within anterior Broca's area does not interfere with phonological processing, supporting claims that areas BA 44 and BA 45 are indeed functionally dissociable. Correspondingly, Devlin et al. (2003) have used TMS to demonstrate disruption of semantic processing when applied over pars triangularis (BA 45) where it borders with pars orbitalis (BA 47).

\section{Experiment 3: Effects of Sham-TMS on Performance}

In the previous experiments, there was an increase in ER when TMS was applied over the opercular region of the IFG. Clearly, the stimulation was having a disruptive effect on performance, but this does not prove that the effect was directly caused by the TMS pulses interacting with the underlying brain tissue. It might be that nonspecific effects associated with the stimulation (e.g., auditory and somatic sensations and the preceding anxiety) could summate to distract attention during critical stages in the performance of the task. Furthermore, all subjects demonstrated a strong TMS-related decrease in RT on the phonological task. For stimulation applied in the delay period the mean RT gain was in the order of $100 \mathrm{msec}$ (Figures 1A and 2), and for stimulation applied at response the gain was approximately $150 \mathrm{msec}$ (Figure 1B). Facilitation effects have been observed on other language tasks (Topper et al., 1998). Such decreases may also have contributed to the increase in ER by promoting premature responses from the subjects (due to a speed-error trade-off). Finally, the decreases in RT might reflect learning-related changes, because the TMS blocks always followed the control blocks.

The purpose of this final experiment was to examine these possible confounding effects in a third group of subjects performing the same tasks but under conditions of sham TMS only. The design of the experiment was largely identical to the previous one except that the TMS coil was angled in such a way that the magnetic field was not directed at the scalp but at right angles to it. This had the effect of exposing the subject to the "clicking" sound associated with rTMS but there were no peripheral somatosensory effects or interactions with neuronal tissue. 


\section{Results and Discussion}

Subjects generally performed faster and more accurately during sham TMS trials than during nonsham trials (Figure 3). The ER decreased slightly for both tasks during sham TMS trials; however, none of the changes in ER were significant and there was no significant difference between the two tasks for either of the sham TMS blocks: Block 1: $t(5)=.67, n s$; Block 2: $t(5)=1.00$, $n s$. For the phonological task, there were significant decreases in RT for both sham TMS blocks, Block 1: $t(5)=-3.65, p<.05$; Block 2: $t(5)=-2.89, p<.05$, and the difference between tasks reached significance for Block 1, $t(5)=-3.99, p=.01$. For the control task, RT decreases were smaller but significant for Block 2 , $t(5)=-5.91, p<.01$. Finally, there were no significant differences in RT between successive sham TMS blocks that might implicate learning effects as a major source of the RT decreases in these experiments: homophones: $t(5)=-1.50, n s$; control: $t(5)=.12, n s$.

Given these results, it is likely that the decrease in RT observed for both tasks can be explained in large part by a nonspecific intersensory facilitation effect (Hershenson, 1963). This effect has been observed before with TMS (e.g., Rushworth, Ellison \& Walsh, 2001; Marzi et al., 1998) and can have the consequence of masking any potential increases in RT directly related to the disruptive effects of TMS on neural function. The results of this experiment also provide further evidence that the significant effects of TMS on response accuracy seen in the previous experiments were not the consequence of a speederror trade-off. Although the subjects in the present group all showed significant decreases in RT during the sham TMS trials, their accuracy was not impaired. It also shows that the noise of the TMS pulses did not distract the subjects during the delay period enough to impair performance.

Lastly, the control data obtained in Experiment 3 allowed a between-subjects comparison of the effects of TMS in the previous experiments. This post hoc analysis confirmed the main finding of a significant decrease in response accuracy for the phonological task when TMS was applied over the posterior part of the IFG, one-way ANOVA $F(2,15)=5.53, p=.02$, corrected, but revealed no significant differences between groups for RT.

\section{DISCUSSION}

Overall, the results of the three experiments provide strong evidence for a disruptive effect of rTMS on phonological memory when applied over the posterior part of Broca's area. Identical stimulation over the same cortical region did not result in an increase in errors on a nonphonologically based memory task. This finding supports the view that the activations shown in this region by neuroimaging studies reflect its contribution to verbal WM strategies such as subvocal articulatory rehearsal (Davachi, Maril, \& Wagner, 2001; Jonides et al., 1998; Cohen et al., 1997; Fiez et al., 1996; Paulesu et al., 1993).

\section{Cognitive Processes}

While the design of this study was intended to minimize the contribution of other cognitive processes, activations in the IFG have also been attributed to learning/encoding
Figure 3. Results of Experiment 3. Changes in percent error rate (\%ER) and RT for sham TMS versus non-TMS trials. Neither the first (A) nor second (B) sham TMS blocks revealed increases in \%ER for either task. However, significant decreases in RT were associated with the clicking sound of the TMS coil (A $+\mathrm{B}$, lower graphs), suggesting the action of intersensory facilitation effects. The standard error of the means is shown by the thin bars. $* p<.05, * * p<.01$.

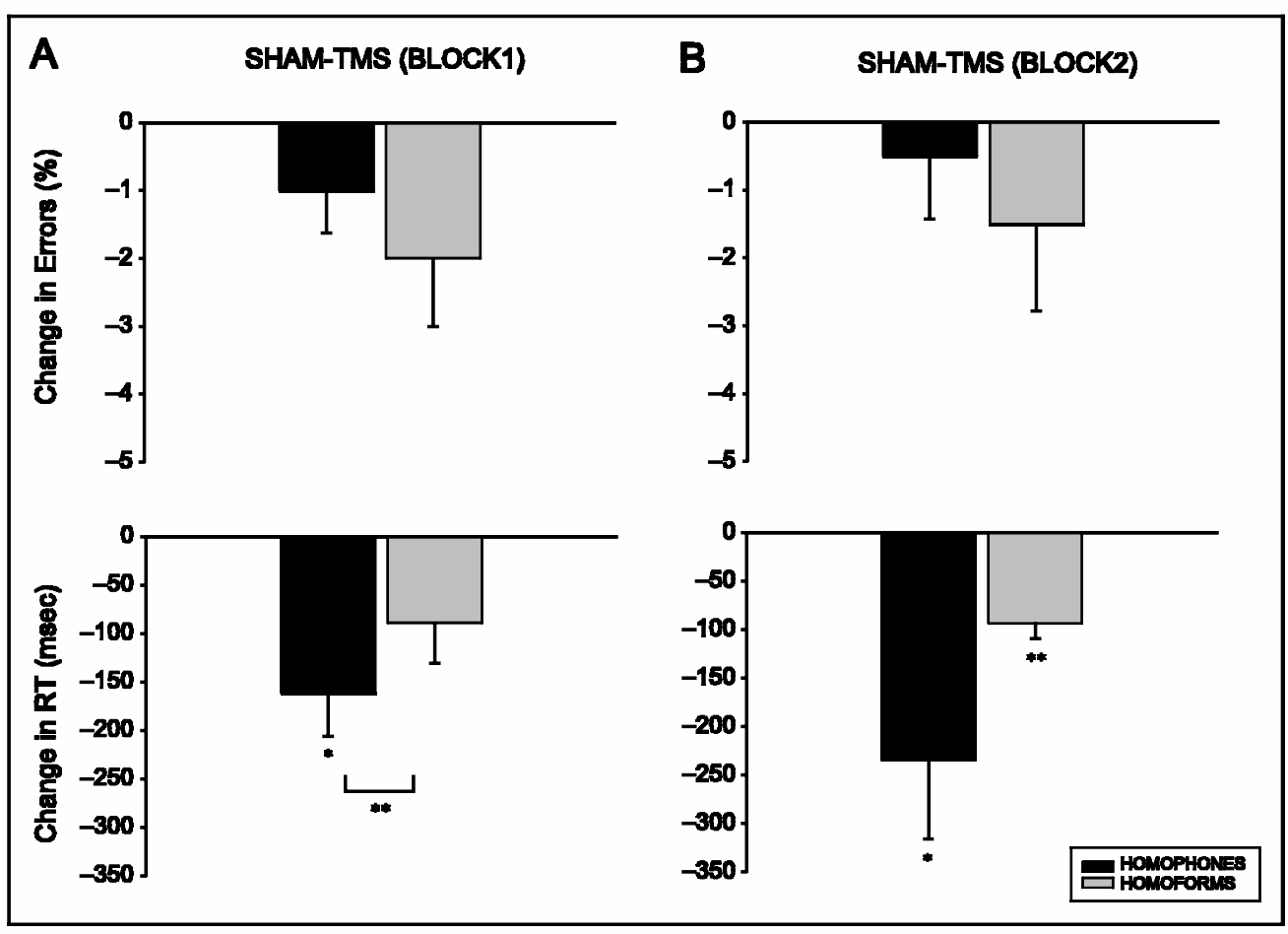


(Davachi et al., 2001; Raichle et al., 1994), phonological decision making (Rumsey et al., 1997), semantic and phonological attention (McDermott et al., 2003), and generative processes (Paulesu et al., 1997). The subjects in the present study were not required to generate paired associates as occurs in phonological verbal fluency tests. Neither were they required to learn anything from one trial to the next, although the small decreases in RT observed over consecutive blocks in Experiment 3 suggests that the subjects were becoming more efficient at executing the task with time.

In Experiment 1, TMS was applied at the decision (response) stage for one block of trials and this caused some subjects to make more errors compared with nonTMS trials. However, the variance of the data was large and no firm conclusions can be drawn from this part of the study. Nonetheless, the possibility that BA 44 is concerned with aspects of phonological decision making warrants further investigation with TMS. In a PET study by Rumsey et al. (1997), subjects were required to decide which of two simultaneously presented nonwords either (a) sounded like a real word or (b) visually matched a real word. The authors found that compared with visual fixation, both tasks produced increases in activity in BA 44, but in a phonological versus orthographic analysis the significant left IFG activations were more widespread, encompassing BA 44, 45, and 46. This indicates that the posterior IFG region tested in the present TMS study is likely to be part of a wider network of inferior frontal regions that are recruited in phonological decision making. In another PET study by Fiez et al. (1996), it was observed that while covert rehearsal of verbal material was associated with left frontal opercular regions, the active maintenance of novel verbal stimuli (as opposed to silent counting) also recruited more dorsolateral prefrontal areas. These and other results suggest that the frontal opercular area (BA 44) is concerned with relatively low-level aspects of phonological processing, perhaps including orthographicto-phonological transformation (Fiez \& Petersen, 1998).

\section{Task Demands}

It could be argued that the reason why TMS interfered with performance of the phonological task but not the control task is that the phonological task was more difficult. On non-TMS trials, the subjects were between 247 msec (Experiment 1) and $181 \mathrm{msec}$ (Experiment 2) slower responding on the phonological task than on the control task. However, it was TMS applied during the delay before presentation of the second item that caused a significant increase in errors. There is no reason to suspect that remembering a word is more difficult than remembering a Korean symbol; indeed, if anything the opposite is probably the case. The reason why it took longer to make the phonological judgment than the visual judgment on presentation of the second item is that in the phonological task the subject had to read the pseudoword and sound it out.

The next question is why the changes in ERs seen in Experiments 1 and 2 were relatively small, although statistically significant. There are several possible reasons why this may be so. First, the phonological task used in this study was somewhat undemanding, using uncomplicated stimuli and short delay lengths. Yet strong activations have been observed in BA 44 when subjects simply remembered the sound of a letter for a subsequent rhyming judgment (Paulesu et al., 1993) or when reading short words was compared with observing a line of asterisks (Georgiewa et al., 2002). However, two independent studies have shown that activity in this region can be influenced either by stimulus difficulty-increasing with the number of syllables or with pseudowords (Chein \& Fiez, 2001) - or by working memory loadincreasing with the number of nontarget items in an $n$-back sequential letter task (Cohen et al., 1997). This suggests that increasing the delay length in the present study, or introducing distractor stimuli during the delay, might have placed more demands on the region such that the effect of the TMS may also have been greater.

TMS was applied for brief (500 msec or less) periods during the delay between stimuli and this may have been insufficient to disrupt ongoing working memory mechanisms for long enough to have a more profound effect. In a more complex design it would be possible to compare the effects of stimulating at different times during the delay, but increasing the duration of the pulse train at the frequency used here would not be possible without exceeding current safety guidelines (Wassermann, 1998). Mottaghy et al. (2002) applied TMS constantly at $4 \mathrm{~Hz}$ during the 30 -sec performance block of a 2-back verbal WM task. As in the present study accuracy, but not RT, was the principal indicator of behavioral impairment related to prefrontal stimulation. The effects were also somewhat greater than in our study and this may have been related to the chronic nature of the TMS or to the longer memory period required $(3 \mathrm{sec})$, or indeed, to the more superior location of the stimulation site used in their study (middle frontal gyrus).

A related issue concerns the uniformity with which the stimulation can be applied and the consistency of its effects in different subjects (Maeda, Keenan, Tormos, Topka, \& Pascual-Leone, 2000; Pascual-Leone et al., 1998). In the present study, the level of stimulation was determined by reference to each subject's motor threshold, but there is a question as to whether this is an appropriate baseline for applying TMS over nonmotor areas of neocortex (Stewart, Walsh, \& Rothwell, 2001). The distance from the scalp to the surface of the prefrontal cortex tends to be greater than the distance from the scalp to the motor cortex and this may be relevant (McConnell et al., 2000). The consequence of this added variability is that it is difficult to equate the 
level of stimulation being applied across subjects without considering this variable depth factor. Ideally, a brief cognitive task should be employed to test for an effect of TMS over the prefrontal cortex, such as is commonly used in TMS studies of the posterior parietal cortex (Ashbridge, Walsh, \& Cowey, 1997).

Finally, despite the significant methodological refinements described in this paper for ensuring the accurate anatomical placement of TMS, loci determined in this way may not correlate precisely with functional regions of interest. For even greater precision it is necessary to obtain functional images, using PET or fMRI, of subjects performing the same tasks that they will subsequently perform with TMS (Mottaghy et al., 2000; Paus et al., 1997). Used in this way, TMS has the potential to provide a powerful method for testing the functional necessity of the cortical activations seen in neuroimaging studies of language, working memory, and other cognitive processes associated with the frontal lobes.

\section{METHODS}

\section{Participants}

Six volunteers ( 3 women) took part in Experiment 1 . They were all right-handed and aged between 18 and 33 (mean 21.7). For Experiment 2 another group of 6 volunteers (3 women) were recruited, none of whom had participated in the previous experiment. The subjects were aged between 21 and 46 (mean 28.5). Five were right-handed and 1 was left-handed but this subject had earlier participated in an ERP study that strongly suggested left hemisphere dominance for language. A further 6 subjects ( 3 women, all right handed) were recruited for Experiment 3 . Their mean age was 23.8 years (range 20 to 33). All the participants in this study were native English speakers and were paid for their participation.

The study was approved by the local institutional ethics board and all subjects gave their informed written consent beforehand. Screening forms were given before testing to assess handedness and to ensure that none of the subjects had any contraindications to TMS. All subjects were informed that they were under no obligation to complete the experiment and could leave at any time.

\section{Task Design}

Subjects were presented with two tasks designed to test either verbal or visual WM. In the verbal WM task (delayed homophone test) subjects were required to silently read a word (e.g., "loops") and to remember the sound of the word during a delay period. They were then presented with an orthographically and phonologically legal pseudoword (e.g., "lupes" or "lirps") and had to decide whether this sounded identical to or different from the preceding stimulus. The stimuli were matched for the number of syllables and the word lists were balanced for word frequency. The visual WM task (delayed homoform test) was identical to the verbal task except that the stimuli consisted of Korean symbols and could only be compared by visual form (none of the subjects could read or speak Korean). Sample trials from both tasks are shown schematically in Figure 4A.

For each task the trials were grouped into blocks of 30 experimental trials preceded by a block of 20 practice trials with error feedback provided on screen. Subjects were seated in an adjustable chair approximately $60 \mathrm{~cm}$ from a 21 -in. VDU on which the stimuli were presented. The first and second fingers of the subject's left hand were placed over the leftmost two keys of a response box (PST, Pittsburgh) labeled "yes" and "no," respectively. Visual stimuli were presented in the center of the screen, black text on a white back-

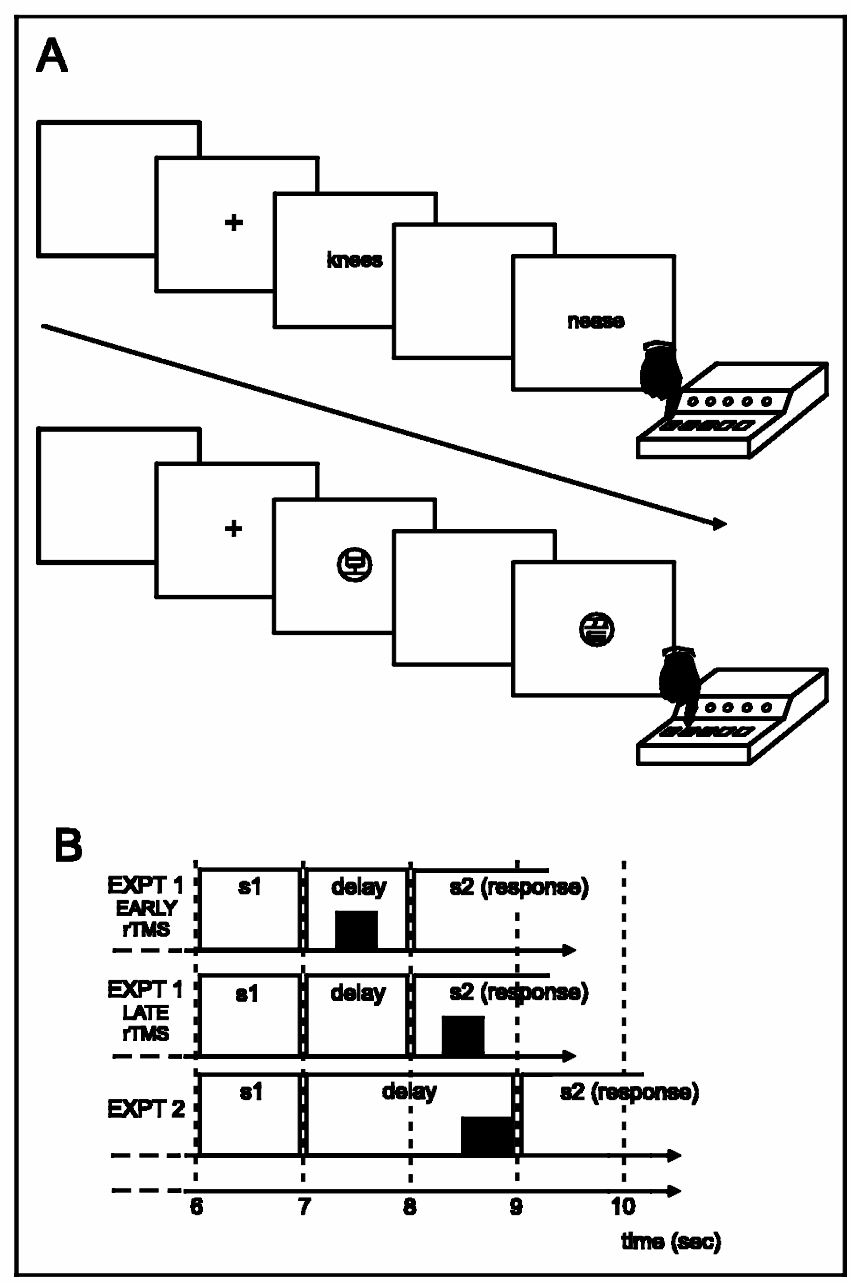

Figure 4. The behavioral tasks used in Experiments 1-3. (A) The upper half of this figure shows a typical trial for the delayed homophone test (phonological matching task) and the lower half shows a typical trial for the delayed homoform test (visual matching task). In both cases, subjects reported their decision by pressing either a "yes" or "no" response key. (B) Time-line showing the onset and offset of the TMS pulse train (solid rectangles) in Experiments 1 and 2. s1 and s2 denote the appearance of the first and second cues. For Experiment 1, TMS was applied either during the delay between the cues or during the response (decision) phase of the trials. 
ground in an 18-point font size. After an intertrial interval lasting $6000 \mathrm{msec}$, a fixation crosshair appeared in the center of the screen for a further $1000 \mathrm{msec}$. This was followed by the appearance of the primary cue (s1 in Figure $4 \mathrm{~B}$ ), in the same position as the crosshair, for $1000 \mathrm{msec}$. This screen was then blanked for a delay period (1000 msec in Experiment 1 and $2000 \mathrm{msec}$ in Experiments 2 and 3), followed immediately by the onset of the secondary cue (s2 in Figure 4B). This stimulus persisted until the subject responded by pressing a key to indicate their choice of either a match or nonmatch between $\mathrm{s} 1$ and $\mathrm{s} 2$.

At the beginning of each task, an instruction page was shown on the screen for the subjects to read. Subjects were then asked if they understood what was required of them. For the homophone test, it was emphasized that they would need to remember the sound of the primary stimulus rather than its form. At the start of each block of each task, subjects were reminded to respond as quickly and accurately as possible. A block design was found to be more agreeable to subjects than a random design because of the increased anxiety inherent in not being able to predict stimulation trials in the latter case. After completion of the practice block, subjects progressed to interleaved blocks of control and rTMS trials. In Experiment 1, stimulation was applied either $300 \mathrm{msec}$ after the start of the delay period or $300 \mathrm{msec}$ after the onset of s2. These parameters were determined from data by Breier, Simos, Zouridakis, and Papanicolaou (1999), who showed peak activations in the left inferior frontal region between 300 and 600 msec after presentation of verbal stimuli in a rhyme-matching task. In Experiment 2, stimulation began $1500 \mathrm{msec}$ after the appearance of s1and lasted until the onset of s2 (Figure $4 \mathrm{~B}$ ). This change was made so that the disruptive effects of the TMS were directed toward the latter part of the delay period in both experiments. We were concerned that if a significant period of time elapsed between the end of the pulse train and the appearance of s2, the subjects might have been able to "refresh" their phonological memory trace for s1 so as to overcome the interference arising from the TMS.

Three of the subjects from each experiment performed the visual WM task first followed by the phonological WM task, and the remaining subjects vice versa. A minimum 2-min rest was enforced between blocks and a further 10-min rest period was interposed between the two tasks. None of the subjects were informed either before or during the experiment as to the expected effects of the TMS on performance and the experimenter avoided referring to the visual WM task as a "control" task.

\section{TMS}

\section{Apparatus}

A MagStim Super-Rapid (MagStim, Whitland, UK) TMS generator connected to a 50-mm figure-of-eight coil provided the stimulation. Previous studies have estimated that such coil designs can produce functionally dissociable effects within a scalp area of $1 \mathrm{~cm}^{2}$ or less (Brasil-Neto, Pascual-Leone, Valls-Sole, Cohen, \& Hallett, 1992). Two such coils (maximum field strength $2.8 \mathrm{~T}$ ) were used interchangeably to offset the effects of overheating the coil windings. The TMS generator was connected to a PC computer running custom software to control the timing of the pulse trains independently of the computer running the behavioral tasks. The task computer simply sent a brief $(1 \mathrm{msec})$ signal to the TMS computer to initiate a pulse train at the required time.

\section{Parameters and Localization}

In an earlier pilot study with 2 TMS experienced subjects ( 1 male, 1 female) we ascertained that TMS-induced speech disruption did not provide reliable localization of Broca's area. From posttest examination of structural MRI scans of the two subjects, the region of maximal disruption lay above the left inferior frontal sulcus, in agreement with Stewart, Meyer, et al. (2001). The site of stimulation for Experiment 1 was therefore estimated with reference to the hand area of the left motor cortex for each individual subject. The level of stimulation used was determined with reference to each subject's active motor threshold (aMT).

A Lycra swimming cap with chin ties to prevent slipping was fitted over the subject's head so that the stimulation sites could be clearly marked with 5-mmdiameter stick-on Velcro spots of different colors. An estimate of the hand area was obtained by moving $5 \mathrm{~cm}$ lateral to the midline at the vertex and $2 \mathrm{~cm}$ rostral. With the center of the coil (also marked by a Velcro spot) placed against the scalp and with its axis lying in the rostrocaudal direction, single pulses of TMS were delivered at various locations until a site was found where it was possible to elicit small movements of the first or middle fingers of the right hand with the muscles in that hand when voluntarily tensed. The minimum level of stimulation required to elicit such movements was then determined by gradually reducing the output power until movements cold no longer be observed.

The target site for stimulation in Experiment 1 was determined by measuring $4 \mathrm{~cm}$ ventral and $3 \mathrm{~cm}$ rostral to the motor cortex site. This was estimated to lie within the opercular region of the inferior frontal gyrus, just below the level of the inferior frontal sulcus. The pilot study referred to above provided structural MRI scans from which the motor site could be located and the target site estimated from cortical features (Figure 5). The TMS coil was held by the experimenter firmly over the target site by aligning the spot marking the center of the coil with the appropriate spot on the subject's head. The handle of the coil was generally aligned in the rostrocaudal 

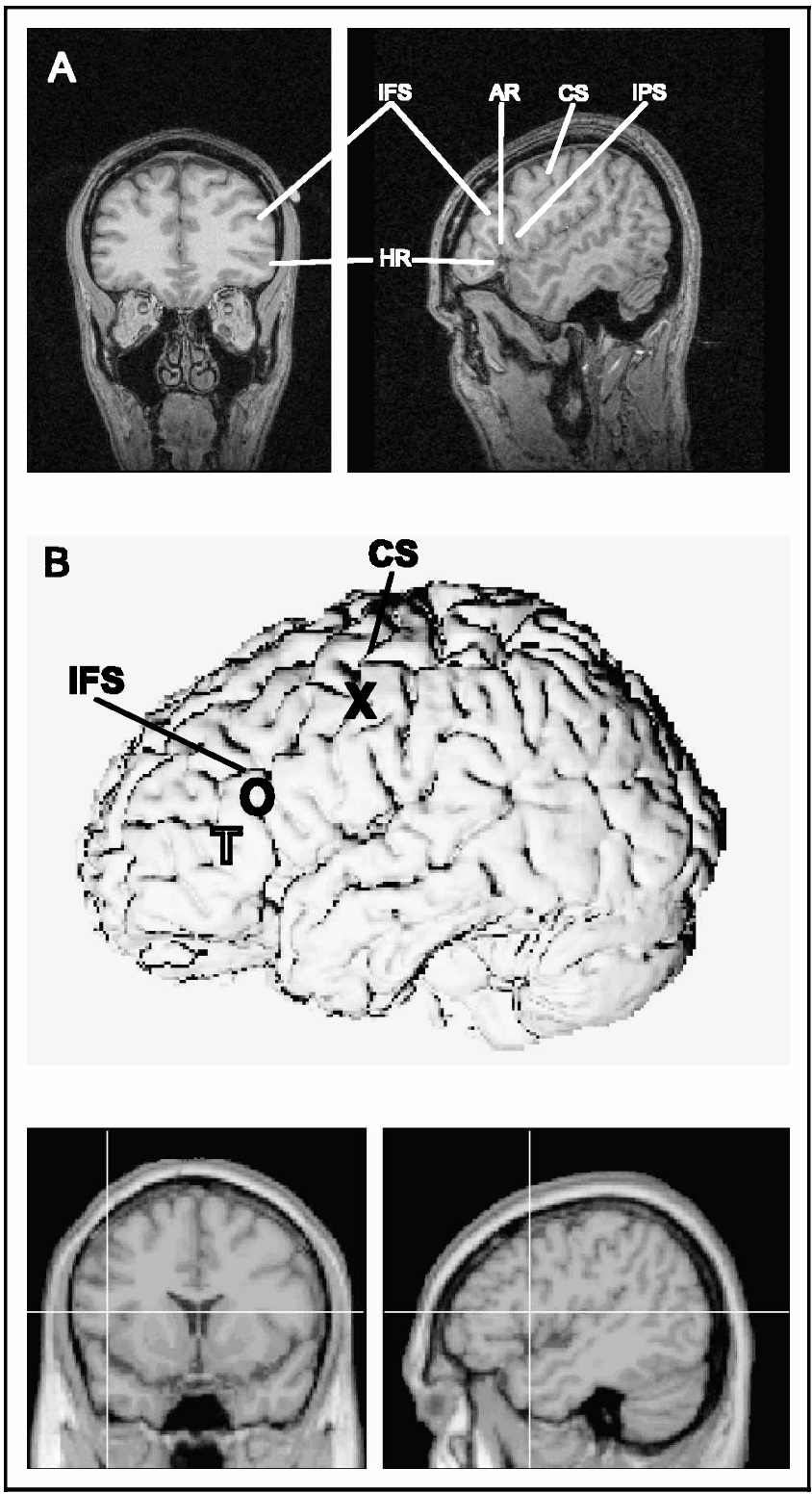

Figure 5. Identification of the TMS sites used in Experiment 1. (A) Coronal (left) and sagittal (left hemisphere) views taken from a structural MRI series of a subject in whom we attempted to ascertain the optimal site for speech disruption. Various cortical features are labeled to indicate the landmarks used to identify Broca's area in this study. $\mathrm{AR} / \mathrm{HR}=$ ascending and horizontal rami of the lateral fissure; $\mathrm{CS}=$ central sulcus; IFS = inferior frontal sulcus; IPS = inferior branch of the precentral sulcus. (B) A surface rendering of the MRI series shown in Figure 5A with symbols superimposed to indicate the TMS target sites. X shows the approximate location of the hand area of the motor cortex. O denotes the posterior (opercular) target for the TMS used in Experiment 1 . The more anterior (triangular, $\mathrm{T}$ ) division of Broca's area was deliberately avoided in this experiment. (C) Coronal (left) and sagittal (left hemisphere) views from a normalized MRI brain showing the location of the activation reported when subjects silently repeat the sound "lah" at $2 \mathrm{~Hz}$ (Paulesu and Passingham, unpublished observations). The coordinates are given in Tailarach space.

plane with some minor $\left(\max 10^{\circ}\right.$ ) adjustments to minimize subject discomfort. If a coil needed to be exchanged due to warming, this was done at the end of a block of trials and the new coil was positioned in the same way as the first.

The level of stimulation used at the target site was variable and limited by the tolerance of each subject to the discomfort associated with rTMS pulse trains. However, the parameters never exceeded the safety guidelines outlined by Wassermann (1998). For Experiment 1 the rTMS pulse train was set at $13.3 \mathrm{~Hz}$ for $375 \mathrm{msec}$, and for Experiment 2 it was set at $10 \mathrm{~Hz}$ for $500 \mathrm{msec}$ because the higher frequency was found to be particularly unpleasant over the more anterior site. Sample trains of rTMS were administered in steps of 5\%, starting from $100 \%$ of aMT, until the subject found the level uncomfortable enough to be potentially distracting during task performance. The mean level of stimulation for the subjects in Experiment 1 was 120\% of aMT (range 100-130\%). These values correspond to approximately $55-75 \%$ of the generator's total power output. For Experiment 2, the levels used were generally lower (mean 110\%, range 100-120\%) but were the same for both sites. This is because TMS at more anterior frontal sites is associated with greater discomfort due to facial muscle activation resulting in jaw movements and forced eye blinks. Two subjects could not tolerate stimulation over this region due to such effects and only received TMS over the posterior target.

\section{"Brainsight"}

For Experiment 2, high-resolution structural MRI scans were obtained for all subjects. A computerized frameless stereotaxy system ("Brainsight," Rogue Research, Montreal) was employed to localize the target sites for stimulation in this experiment. This method greatly improves the anatomical localization for this kind of study and can be used to help target functional brain activity recorded before the TMS session. Briefly, reflective markers attached to the subject's head are monitored by infrared sensitive cameras to ensure that movements of the subject's head are correlated in real time with the MRI data set. The MRI data are coregistered to the head by reference to easily visible cranial landmarks. Reflective markers attached to the TMS coil allow the system to track the position of the coil relative to the head MRI and optimal coil positioning can be determined for each target site.

Identification of the appropriate sulci and gyri that assist in defining the classic Broca's area was made by reference to the atlas of Duvernoy, Bourgouin, Cabanis, and Cattin (1999) and an MRI examination technique developed by Bacon-Moore, Crosson, Gokay, Leonard, and Foundas (2000). The boundary between the pars opercularis and pars triangularis divisions of Broca's area was a line drawn along the anterior ascending ramus of the sylvian fissure (Figure 5A). It should be noted that this method provides a limited anatomical definition of areas that can only be precisely denoted with further 

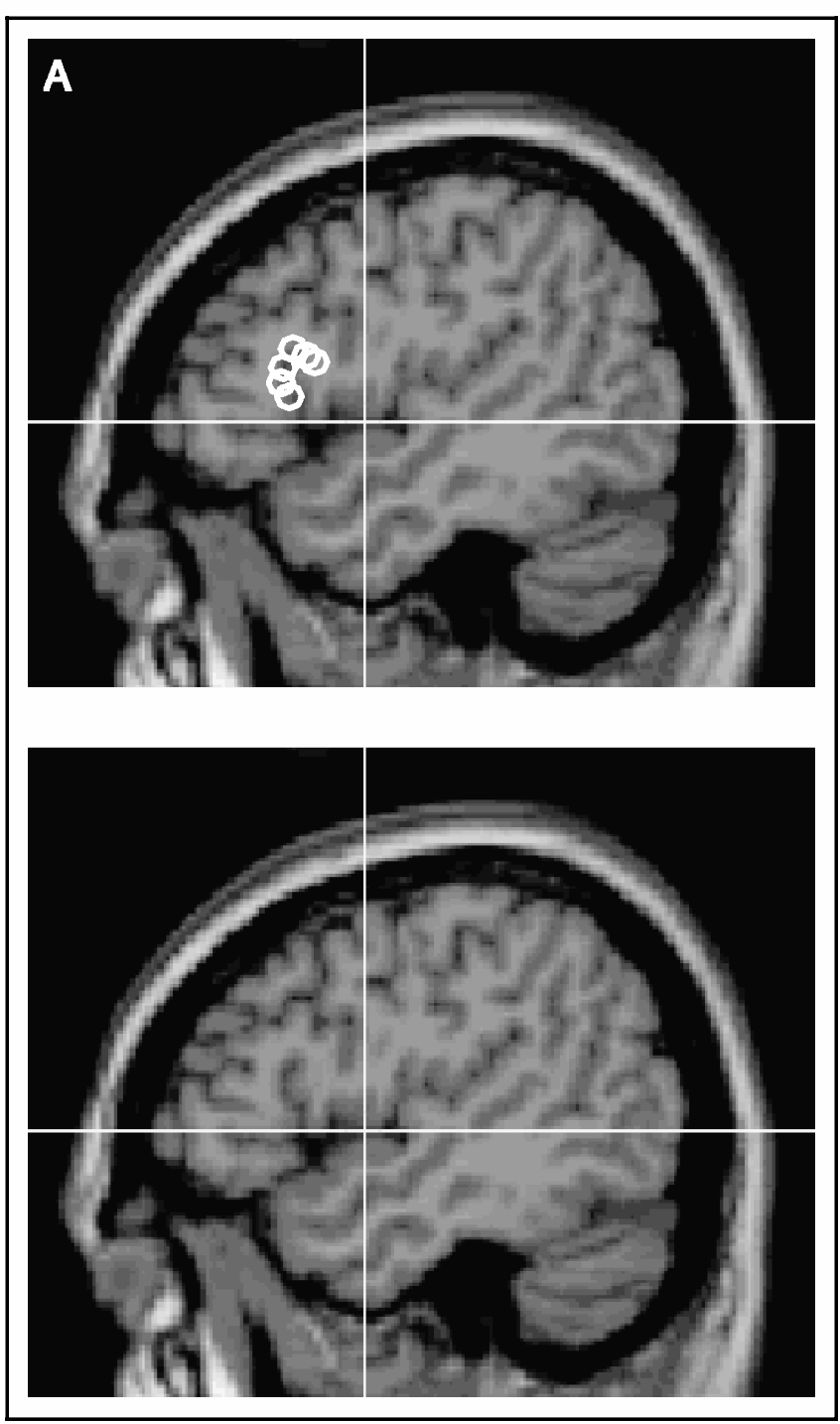

Figure 6. Identification of the TMS sites used in Experiment 2. (A) The locations (white circles) of the stimulation points in all six subjects for TMS applied over posterior Broca's area (pars opercularis). The points were determined by reference to MRI scans of each individual subject but are plotted here onto a left hemisphere sagittal view $(x=-48)$ from a standard brain MRI (MNI template). All the sites lay between $y=+11$ to +21 and $z=+6$ to +20 , thus falling within the higher probability contour maps for the pars opercularis defined in Tomaiuolo et al. (1999). The crosshairs show the position of the anterior commissure $(y=0)$. (B) Location of stimulation points for TMS targeted over the anterior part of Broca's area (pars triangularis/ orbitalis). These points lie between $y=+24$ and $+37, z=-7$ and +3 .

reference to cortical cytoarchitecture (Amunts et al., 1999). The sites chosen for stimulation in the six subjects are shown in Figure 6.

\section{Sham TMS}

Experiment 3 was run in identical fashion to Experiment 2 except that sham TMS stimulation was given by orienting the center of the coil $90^{\circ}$ from its normal position tangential to the scalp. This reproduced the acoustic stimulation associated with the TMS trains used in the previous experiments, but did not induce the peripheral somatosensory effects associated with TMS over prefrontal sites. The subjects were made aware before the experiment began that they would not be receiving any invasive stimulation.

\section{Data Analysis}

Error rate and RT were chosen as dependent variables for analysis of the effects of TMS on task performance. Repeated measures $t$ tests were performed on the unprocessed data to show any significant differences between blocks of TMS (or sham TMS) and non-TMS trials for each condition. To examine differences between the two tasks, similar tests were performed on normalized data obtained by subtracting the values for non-TMS trials from those of the TMS trials. In addition, one-sample $t$ tests were used to reveal if any of the changes in ER or RT for each task differed significantly from the baseline (i.e., no change). Finally, a series of single-factor ANOVAs, corrected for multiple comparisons (Tukey test) were performed on the normalized data to examine differences in accuracy and RT between all three experiments.

\section{Acknowledgments}

This work was supported by a grant from the Wellcome Trust (UK).

Reprint requests should be sent to P. D. Nixon, Department of Experimental Psychology, University of Oxford, South Parks Road, Oxford OX1 3UD, UK, or via e-mail: philip.nixon@psy. ox.ac.uk.

\section{REFERENCES}

Amunts, K., Schleicher, A., Burgel, U., Mohlberg, H., Uylings, H. B. M., \& Zilles, K. (1999). Broca's region revisited:

Cytoarchitecture and intersubject variability. The Journal of Comparative Neurology, 412, 319-341

Ashbridge, E., Walsh, V., \& Cowey, A. (1997). Temporal aspects of visual search studied by transcranial magnetic stimulation. Neuropsychologia, 35, 1121-1131.

Bacon Moore, A., Crosson, B., Gokcay, D., Leonard, C. M., \& Foundas, A. L. (2000). Defining pars triangularis. Society For Neuroscience Abstracts, 26, 464.15.

Bartres-Faz, D., Pujol, J., Deus, J., Tormos, J. M., Keenan, J. P., \& Pascual-Leone, A. (1999). Identification of the brain areas from which TMS induces speech arrest in normal subjects. Neurolmage, 9, S1051.

Brasil-Neto, J. P., Pascual-Leone, A., Valls-Sole, J., Cohen, L. G., \& Hallett, M. (1992). Focal transcranial magnetic stimulation and response bias in a forced-choice task. Journal of Neurology, Neurosurgery and Psychiatry, 55, 964-966.

Breier, J. I., Simos, P. G., Zouridakis, G., \& Papanicolaou, A. C. (1999). Temporal course of regional brain activation associated with phonological processing. Journal of Clinical and Experimental Neuropsychology, 21, 465-476.

Chein, J. M., \& Fiez, J. A. (2001). Dissociation of verbal working memory system components using a delayed serial recall task. Cerebral Cortex, 11, 1003-1014. 
Cohen, J. D., Perlstein, W. M., Braver, T. S., Nystrom, L. E., Noll, D. C., Jonides, J., \& Smith. E. E. (1997). Temporal dynamics of brain activation during a working memory task. Nature, 386, 604-607.

Davachi, L., Maril, A., \& Wagner, A. D. (2001). When keeping in mind supports later bringing to mind: Neural markers of phonological rehearsal predict subsequent remembering. Journal of Cognitive Neuroscience, 13, 1059-1070.

Demonet, J. F., Chollet, F., Ramsay, S., Cardebat, D., Nespoulous, J. D, \& Wise, R. (1992). The anatomy of phonological and semantic processing in normal subjects. Brain, 115, 1753-1768.

Devlin, J. T., Matthews, P. M., \& Rushworth, M. F. S. (2003). Semantic processing in the left inferior prefrontal cortex: A combined fMRI and TMS study. Journal of Cognitive Neuroscience, 15, 71-84.

Duvernoy, H. M., Bourgouin, P., Cabanis, E. A., \& Cattin, F. (1999). The buman brain: Surface, three-dimensional sectional anatomy with MRI and blood supply. New York: Springer-Verlag.

Epstein, C. M., Lah, J. J., Meador, K., Weissman, J. D., Gaitan, L. E., \& Dihenia, B. (1996). Optimum stimulus parameters for lateralized suppression of speech with magnetic brain stimulation. Neurology, 47, 1590-1593.

Epstein, C. M., Meador, K., Loring, D. W., Wright, R. J., Weissman, J. D., Sheppard, S., Lah, J. J., Puhalovich, F., Gaitan, L., \& Davey, K. R. (1999). Localization and characterization of speech arrest during transcranial magnetic stimulation. Clinical Neurophysiology, 110, 1073-1079.

Fiez, J. A., \& Petersen, S. E. (1998). Neuroimaging studies of word reading. Proceedings of the National Academy of Sciences, U.S.A., 95, 914-921.

Fiez, J. A., Raife, E. A., Balota, D. A., Scwarz, J. P., Raichle, M. E., \& Petersen, S. E. (1996). A positron emission tomography study of the short-term maintenance of verbal information. Journal of Neuroscience, 16, 808-822.

Flitman, S. S., Grafman, J., Wasserman, M. D., Cooper, B. A., O'Grady, J., Pascual-Leone, A., \& Hallett, M. (1998).

Linguistic processing during repetitive transcranial magnetic stimulation. Neurology, 50, 175-181.

Georgiewa, P., Rzanny, R., Gaser, C., Gerhard, U. J., Vieweg, U., Mentzel, H. J., Kaiser, W. A., \& Blanz, B. (2002). Phonological processing in dyslexic children: A study combining functional imaging and event related potentials. Neuroscience Letters, 318, 5-8.

Grezes, J., \& Decety, J. (2001). Functional anatomy of execution, mental simulation, observation, and verb generation of actions: A meta-analysis. Human Brain Mapping, 12, 1-19.

Hershenson, M. (1963). Reaction time as a measure of intersensory facilitation. Journal of Experimental Psychology, 63, 289-293.

Jahanshahi, M., \& Rothwell, J. (2000). Transcranial magnetic stimulation studies of cognition: An emerging field. Experimental Brain Research, 13, 1-9.

Jonides, J., Schumacher, E. H., Smith, E. E., Koeppe, R. A., Awh, E., Reuter-Lorenz, P. A., Marshuetz, C., \&Willis, C. R. (1998). The role of the parietal cortex in verbal working memory. The Journal of Neuroscience, 18, 5026-5034.

Maeda, F., Keenan, J., Tormos, J. M., Topka, H., \& Pascual-Leone, A. (2000). Interindividual variability of the modulatory effects of repetitive transcranial magnetic stimulation on cortical excitability. Experimental Brain Research, 133, 425-430.

Marzi, C. A., Miniussi, C., Maravita, A., Bertolasi, L., Zanette, G., Rothwell, J. C., \& Sanes, J. N. (1998). Transcranial magnetic stimulation selectively impairs interhemispheric transfer of visuo-motor information in humans. Experimental Brain Research, 118, 435-438.

McConnell, K. A., Nahas, Z., Kozel, F. A., Lorberbaum, J. P., Shastri, A., Bohning, D. E., \& George, M. S. (2000). The TMS motor threshold depends on the distance from coil to underlying cortex: A replication in healthy adults. Neurolmage, 11, S535.

McDermott, K. B., Petersen, S. E., Watson, J. M., \& Ojemann, J. G. (2003). A procedure for identifying regions preferentially activated by attention to semantic and phonological relations using functional magnetic resonance imaging. Neuropsychologia, 41, 293-303.

Mottaghy, F. M., Doring, T., Muller-Gartner, H. W., Topper, R., \& Krause, B. J. (2002). Bilateral parieto-frontal network for verbal working memory: An interference approach using repetitive transcranial magnetic stimulation (rTMS). European Journal of Neuroscience, 16, 1627-1632.

Mottaghy, F. M., Krause, B. J., Kemna, L. J., Topper, R., Tellmann, L., Beu, M., Pascual-Leone, A., \& Muller-Gartner, H. W. (2000). Modulation of the neuronal circuitry subserving working memory in healthy human subjects by repetitive transcranial magnetic stimulation. Neuroscience Letters, 280, 167-170.

Pascual-Leone, A., Tarazona, F., Keenan, J., Tormos, J. M., Hamilton, R., \& Catala, M. D. (1999). Transcranial magnetic stimulation and neuroplasticity. Neuropsychologia, 37, 207-217.

Pascual-Leone, A., Tourmos, J. M., Keenan, J., Tarazona, F., Canete, C., \& Catala, M. D. (1998). Study and modulation of cortical excitability with transcranial magnetic stimulation. Journal of Clinical Neurophysiology, 15, 333-343.

Paulesu, E., Frith, C. D., \& Frackowiak, R. S. J. (1993). The neural correlates of the verbal component of working memory. Nature, 362, 342-345.

Paulesu, E., Goldacre, B., Scifo, P., Cappa, S. F., Gilardi, M. C., Castiglioni, I., Perani, D., \& Fazio, F. (1997). Functional heterogeneity of left inferior frontal cortex revealed by fMRI. NeuroReport, 8, 2011-2016.

Paus, T., Jech, R., Thompson, C. J., Comeau, R., Peters, T., \& Evans, A. C. (1997). Transcranial magnetic stimulation during positron emission tomography: A new method for studying connectivity of the human cerebral cortex. The Journal of Neuroscience, 17, 3178-3184.

Poldrack, R. A., Wagner, A. D., Prull, M. W., Desmond, J. E., Glover, G. H., \& Gabrielli, J. D. (1999). Functional specialization for semantic and phonological processing in the left inferior prefrontal cortex. Neurolmage, 10, 15-35.

Price, C. J., Indefrey, P., \& Turennout, M. (1999). The neural architecture underlying the processing of written and spoken word forms. In C. M. Brown \& P. Hagoort (Eds.), The neurocognition of language (pp. 211-240). Oxford: OUP.

Price, C. J., Wise, R. J. S., Warburton, E. A., Moore, C. J., Howard, D., Patterson, K., Frackowiak, R. S., \& Friston, K. J. (1996). Hearing and saying: The functional neuro-anatomy of auditory word processing. Brain, 119, 919-931.

Raichle, M. E., Fiez, J. A., Videen, T. O., MacLeod, A. K., Pardo, J. V., Fox, P. T., \& Petersen, S. E. (1994). Practice related changes in human brain functional anatomy during non-motor learning. Cerebral Cortex, 4, 8-26.

Rumsey, J. M., Horwitz, B., Donohue, B. C., Nace, K., Maisog, J. M., \& Andreason, P. (1997). Phonological and orthographic components of word recognition. A PET-rCBF study. Brain, 120, 739-759.

Rushworth, M. F. S., Ellison, A., \& Walsh, V. (2001). Complementary localization and lateralization of orienting and motor attention. Nature Neuroscience, 4, 656-661.

Shapiro, K. A., Pascual-Leone, A., Mottaghy, F. M., Gangitano, M., \& Caramazza, A. (2001). Grammatical distinctions in the 
left frontal cortex. Journal of Cognitive Neuroscience, 13, $713-720$.

Signoret, J. L., Castaigne, P., Lhermitte, F., Abelanet, R., \& Lavorel, P. (1984). Rediscovery of Leborgne's brain: Anatomical description with CT scan. Brain E Language, 22, 303-319.

Stewart, L., Meyer, B. U., Frith, U., \& Rothwell, J. (2001). Left posterior BA37 is involved in object recognition: A TMS study. Neuropsychologia, 39, 1-6.

Stewart, L., Walsh, V., Frith, U., \& Rothwell, J. (2001). TMS produces two dissociable types of speech disruption. NeuroImage, 13, 472-478.

Stewart, L., Walsh, V., \& Rothwell, J. (2001). Motor and phosphene thresholds: A transcranial magnetic stimulation correlation study. Neuropsychologia, 39, 415-419.

Tomaiuolo, F., MacDonald, J. D., Caramanos, Z., Posner, G., Chiavaras, M., Evans, A. C., \& Petrides, M. (1999). Morphology, morphometry and probability mapping of the pars opercularis of the inferior frontal gyrus: An in vivo MRI analysis. European Journal of Neuroscience, 11, 3033-3046.
Topper, R., Mottaghy, F. M., Brugmann, M., Noth, J., \& Huber, W. (1998). Facilitation of picture naming by focal transcranial magnetic stimulation of Wernike's area. Experimental Brain Research, 121, 371-378.

Walsh, V., \& Cowey, A. (2000). Transcranial magnetic stimulation and cognitive neuroscience. Nature Reviews, 1 , 73-79.

Warburton, E., Wise, R. J., Price, C. J., Weiller, C., Hadar, U., Ramsay, S., \& Frackowiak, R. S. J. (1996). Studies with positron emission tomography of noun and verb retrieval in normal subjects. Brain, 119, 159-180.

Wassermann, E. M. (1998). Risk and safety of repetitive transcranial magnetic stimulation: Report and suggested guidelines from the International Workshop on the Safety of Repetitive Transcranial Magnetic Stimulation, June 5-7, 1996. Electroencephalography and Clinical Neurophysiology, 108, 1-16.

Willmes, K., \& Poeck, K. (1993). To what extent can aphasic syndromes be localized? Brain, 116, 1527-1540.

Zattore, R. J., Evans, A. C., Meyer, E., \& Gjedde, A. (1992). Lateralisation of phonetic and pitch discrimination in speech processing. Science, 256, 846-849. 